K. Matsumoto

Nagoya Math. J.

Vol. 165 (2002), 105-112

\title{
ON THE COHOMOLOGICAL COMPLETENESS OF $q$-COMPLETE DOMAINS WITH CORNERS
}

\author{
KAZUKO MATSUMOTO
}

\begin{abstract}
We prove the vanishing and non-vanishing theorems for an intersection of a finite number of $q$-complete domains in a complex manifold of dimension $n$. When $q$ does not divide $n$, it is stronger than the result naturally obtained by combining the approximation theorem of Diederich-Fornaess for $q$ convex functions with corners and the vanishing theorem of Andreotti-Grauert for $q$-complete domains. We also give an example which implies our result is best possible.
\end{abstract}

\section{Introduction}

Let $D$ be a complex manifold of dimension $n$ and let $q$ be an integer with $1 \leq q \leq n$. A continuous function from $D$ to $\mathbb{R}$ is called $q$-convex with corners if it is locally a maximum of a finite number of $q$-convex functions. In $[\mathrm{D}-\mathrm{F}]$ Diederich-Fornaess proved that every $q$-convex function with corners defined on $D$ can be approximated by $\widetilde{q}$-convex functions whole on $D$, where $\widetilde{q}:=n-[n / q]+1$ and $[x]$ denotes the integral part of $x$. They moreover showed that the number $\widetilde{q}$ is best possible for any $(n, q)$, i.e., there exist an open subset $D$ in $\mathbb{C}^{n}$ and a finite number of $q$-convex functions $\varphi_{1}, \ldots, \varphi_{s}$ defined on $D$ such that the function $\varphi:=\max \left\{\varphi_{1}, \ldots, \varphi_{s}\right\}$ cannot be approximated by $(\widetilde{q}-1)$-convex functions.

A complex manifold $D$ is called $q$-complete (resp. $q$-complete with corners) if $D$ has an exhaustion function which is $q$-convex (resp. $q$-convex with corners) on $D$. Combining the above theorem of Diederich-Fornaess with the theorem of Andreotti-Grauert ([A-G]) it follows at once that if $D$ is $q$-complete with corners then $D$ is cohomologically $\widetilde{q}$-complete.

Now the following problem arises naturally.

PROBLEM. Is there a complex manifold which is q-complete with corners but not cohomologically $(\widetilde{q}-1)$-complete?

Received May 15, 2001.

2000 Mathematics Subject Classification: 32F10. 
It is easy to find such examples if $q$ divides $n$ (cf. [S-V], [E-S], [M-1] and $[\mathrm{M}-2])$. However, it seems that such an example is still unknown if $q$ does not divide $n$.

The purpose of this article is to prove the following.

TheOREM. Let $M$ be a complex manifold of dimension $n$ and let $D_{1}, \ldots, D_{t}$ be $q$-complete open subsets in $M$. Let $\mathcal{F}$ be a coherent analytic sheaf on $M$ such that $H^{n}(M, \mathcal{F})=0$. Then

$$
H^{j}\left(D_{1} \cap \cdots \cap D_{t}, \mathcal{F}\right)=0 \quad \text { if } \quad j \geq \widehat{q}_{t} .
$$

Here

$$
\widehat{q}_{t}:=\min \{\widehat{q}, t(q-1)+1\}
$$

and

$$
\widehat{q}:=n-\left[\frac{n-1}{q}\right]=\left\{\begin{array}{lll}
\widetilde{q} & \text { if } & q \mid n \\
\widetilde{q}-1 & \text { if } & q \nmid n .
\end{array}\right.
$$

Moreover, the number $\widehat{q}_{t}$ in the above theorem is best possible for any $(n, q, t)$. In particular, for any $(n, q)$ there exist a finite number of $q$-complete open subsets $D_{1}, \ldots, D_{s}$ in $\mathbb{C}^{n}$ such that $H^{\widehat{q}-1}\left(D_{1} \cap \cdots \cap D_{s}, \mathcal{O}\right) \neq 0$, where $\mathcal{O}$ denotes the sheaf of germs of holomorphic functions on $\mathbb{C}^{n}$ (see $\S 3$ ).

The author, in general, does not know whether the cohomologically $\widehat{q}-$ complete set $D_{1} \cap \cdots \cap D_{t}$ in the above theorem is $\widehat{q}$-complete, i.e., it has a $\widehat{q}$-convex exhaustion function, even in the case $M=\mathbb{C}^{n}$.

\section{$\S 1$. The key proposition}

First we show the following proposition which is a key step to prove Theorem.

Proposition 1. Let $M$ be a topological space, let $\left\{D_{1}, \ldots, D_{t}\right\}$ be a family of open subsets in $M$ and let $\mathcal{S}$ be a sheaf of Abelian groups on $M$. Let $p \in \mathbb{N}$ be fixed and suppose that for any $k$ with $1 \leq k \leq t-1$ the family $\left\{D_{1}, \ldots, D_{t}\right\}$ satisfies the condition

$C(k, p)$

$$
H^{j}\left(D_{i_{1}} \cap \cdots \cap D_{i_{k}}, \mathcal{S}\right)=0
$$

$$
\text { for all } j \geq p \text { and all } i_{1}, \ldots, i_{k} \in\{1,2, \ldots, t\} \text {. }
$$

Then

(1) $\quad H^{j}\left(D_{1} \cap \cdots \cap D_{t}, \mathcal{S}\right) \cong H^{j+t-1}\left(D_{1} \cup \cdots \cup D_{t}, \mathcal{S}\right) \quad$ if $\quad j \geq p$;

(2) $\quad H^{p-1}\left(D_{1} \cap \cdots \cap D_{t}, \mathcal{S}\right) \rightarrow H^{p+t-2}\left(D_{1} \cup \cdots \cup D_{t}, \mathcal{S}\right)$ 
Remark. The family $\left\{D_{1}, \ldots, D_{t}\right\}$ satisfies the condition $C(k, p)$ for all $k$ with $1 \leq k \leq t-1$ if it satisfies only $C(t-1, p)$.

Proof of Proposition 1. We shall prove the proposition by induction on $t \in \mathbb{N}$.

Step 1. When $t=1,(1)$ and (2) are trivial.

Step 2. When $t \geq 2$, it follows by Mayer-Vietories that the sequence

$$
\begin{aligned}
H^{j}\left(D_{1} \cap\right. & \left.\cdots \cap D_{t-1}, \mathcal{S}\right) \oplus H^{j}\left(D_{t}, \mathcal{S}\right) \\
& \longrightarrow H^{j}\left(\left(D_{1} \cap \cdots \cap D_{t-1}\right) \cap D_{t}, \mathcal{S}\right) \\
& \longrightarrow H^{j+1}\left(\left(D_{1} \cap \cdots \cap D_{t-1}\right) \cup D_{t}, \mathcal{S}\right) \\
& \longrightarrow H^{j+1}\left(D_{1} \cap \cdots \cap D_{t-1}, \mathcal{S}\right) \oplus H^{j+1}\left(D_{t}, \mathcal{S}\right)
\end{aligned}
$$

is exact for each $j$. Since $\left\{D_{1}, \ldots, D_{t}\right\}$ satisfies $C(t-1, p)$ and $C(1, p)$ by assumption, we have

$$
H^{j}\left(D_{1} \cap \cdots \cap D_{t-1}, \mathcal{S}\right)=H^{j}\left(D_{t}, \mathcal{S}\right)=0 \quad \text { if } \quad j \geq p .
$$

Therefore, if we put $E_{i}:=D_{i} \cup D_{t}$ for $i=1,2, \ldots, t-1$, then

(3) $\quad H^{j}\left(D_{1} \cap \cdots \cap D_{t}, \mathcal{S}\right) \cong H^{j+1}\left(E_{1} \cap \cdots \cap E_{t-1}, \mathcal{S}\right) \quad$ if $\quad j \geq p$;

(4) $\quad H^{p-1}\left(D_{1} \cap \cdots \cap D_{t}, \mathcal{S}\right) \rightarrow H^{p}\left(E_{1} \cap \cdots \cap E_{t-1}, \mathcal{S}\right)$.

In particular, this means that the proposition holds in the case $t=2$.

Step 3. When $t \geq 3$, we assume that the proposition has been proved for $1,2, \ldots, t-1$. We first show the following.

LEMMA 1. Under the above situation, the family $\left\{E_{1}, \ldots, E_{t-1}\right\}$ satisfies the condition $C(t-2, p+1)$.

Proof. We shall prove by induction that for any $l$ with $1 \leq l \leq t-2$ the family $\left\{E_{1}, \ldots, E_{t-1}\right\}$ satisfies the condition

$C(l, p+1)$

$$
H^{j+1}\left(E_{i_{1}} \cap \cdots \cap E_{i_{l}}, \mathcal{S}\right)=0
$$

for all $j \geq p$ and all $i_{1}, \ldots, i_{l} \in\{1,2, \ldots, t-1\}$. 
By the assumption of the proposition $\left\{D_{1}, \ldots, D_{t}\right\}$ satisfies $C(t-1, p)$ and particularly $C(1, p)$ and $C(2, p)$. Since the proposition holds in the case $t=2$ we have

$$
\begin{aligned}
H^{j+1}\left(E_{i_{1}}, \mathcal{S}\right) & =H^{j+1}\left(D_{i_{1}} \cup D_{t}, \mathcal{S}\right) \\
& \cong H^{j}\left(D_{i_{1}} \cap D_{t}, \mathcal{S}\right)=0 \quad \text { if } \quad j \geq p .
\end{aligned}
$$

Therefore, $\left\{E_{1}, \ldots, E_{t-1}\right\}$ satisfies $C(1, p+1)$.

Next let $2 \leq l \leq t-2$ and assume that the lemma has been proved for all $m$ with $1 \leq m \leq l-1$. Then the family $\left\{E_{i_{1}}, \ldots, E_{i_{l}}\right\}$, where $i_{1}, \ldots, i_{l} \in\{1,2, \ldots, t-1\}$, also satisfies the condition $C(m, p+1)$ for all $m$ with $1 \leq m \leq l-1$. Moreover, since $\left\{E_{1}, \ldots, E_{t-1}\right\}$ satisfies $C(l-1, p+1)$ by the inductive hypothesis and since the proposition holds for $l$,

$$
\begin{aligned}
H^{j+1}\left(E_{i_{1}}\right. & \left.\cap \cdots \cap E_{i_{l}}, \mathcal{S}\right) \\
& \cong H^{(j+1)+l-1}\left(E_{i_{1}} \cup \cdots \cup E_{i_{l}}, \mathcal{S}\right) \\
& =H^{j+(l+1)-1}\left(D_{i_{1}} \cup \cdots \cup D_{i_{l}} \cup D_{t}, \mathcal{S}\right) \quad \text { if } \quad j \geq p .
\end{aligned}
$$

On the other hand, $\left\{D_{1}, \ldots, D_{t}\right\}$ satisfies $C(l, p)$ and $C(l+1, p)$ because $l+1 \leq t-1$. Since the proposition holds for $l+1$,

$$
\begin{aligned}
& H^{j+(l+1)-1}\left(D_{i_{1}} \cup \cdots \cup D_{i_{l}} \cup D_{t}, \mathcal{S}\right) \\
& \quad \cong H^{j}\left(D_{i_{1}} \cap \cdots \cap D_{i_{l}} \cap D_{t}, \mathcal{S}\right)=0 \quad \text { if } \quad j \geq p .
\end{aligned}
$$

Hence we obtain

$$
H^{j+1}\left(E_{i_{1}} \cap \cdots \cap E_{i_{l}}, \mathcal{S}\right)=0 \quad \text { if } \quad j \geq p,
$$

which proves that $\left\{E_{1}, \ldots, E_{t-1}\right\}$ satisfies $C(l, p+1)$ for all $l$ with $1 \leq l \leq$ $t-2$.

End of Proof of Proposition 1. If $t \geq 3$ and if $\left\{D_{1}, \ldots, D_{t}\right\}$ satisfies $C(t-1, p)$ then $\left\{E_{1}, \ldots, E_{t-1}\right\}$ satisfies $C(t-2, p+1)$, where $E_{i}:=D_{i} \cup D_{t}$ for $i=1,2, \ldots, t-1$. Therefore, by the inductive hypothesis, we have

$$
\begin{aligned}
& H^{j+1}\left(E_{1} \cap \cdots \cap E_{t-1}, \mathcal{S}\right) \cong H^{j+t-1}\left(E_{1} \cup \cdots \cup E_{t-1}, \mathcal{S}\right) \quad \text { if } \quad j \geq p \\
& H^{p}\left(E_{1} \cap \cdots \cap E_{t-1}, \mathcal{S}\right) \rightarrow H^{p+t-2}\left(E_{1} \cup \cdots \cup E_{t-1}, \mathcal{S}\right) .
\end{aligned}
$$

Notice here that $E_{1} \cup \cdots \cup E_{t-1}=D_{1} \cup \cdots \cup D_{t}$. Then we can obtain (1) and (2) by (3), (4), (5) and (6).

This completes the proof of the proposition. 


\section{§2. Proof of Theorem}

Let $M$ be a complex manifold of dimension $n$, let $D_{1}, \ldots, D_{t}$ be $q$ complete open subsets in $M$ and let $\mathcal{F}$ be a coherent analytic sheaf on $M$ such that $H^{n}(M, \mathcal{F})=0$.

Since the intersection $D_{1} \cap \cdots \cap D_{t}$ is $q$-complete with corners it follows from the theorem of Diederich-Fornaess and the theorem of AndreottiGrauert that

$$
H^{j}\left(D_{1} \cap \cdots \cap D_{t}, \mathcal{F}\right)=0 \quad \text { if } \quad j \geq \widetilde{q}_{t} .
$$

Here $\widetilde{q}_{t}:=\min \{\widetilde{q}, t(q-1)+1\}$ and $\widetilde{q}:=n-[n / q]+1$.

We put

$$
\widehat{q}:=n-\left[\frac{n-1}{q}\right]=\left\{\begin{array}{lll}
\widetilde{q} & \text { if } & q \mid n \\
\widetilde{q}-1 & \text { if } & q \nmid n .
\end{array}\right.
$$

For the proof of Theorem it is enough to prove the following.

LEMMA 2. Under the above situation,

$$
H^{j}\left(D_{1} \cap \cdots \cap D_{t}, \mathcal{F}\right)=0 \quad \text { if } \quad j \geq \widehat{q} .
$$

Proof. We put $m:=[n / q]$ and $r:=n-m q$. Then $n=m q+r$ and $0 \leq r \leq q-1$. We shall prove the lemma by induction on $t \in \mathbb{N}$.

First if $t \leq m$,

$$
t(q-1)+1 \leq m(q-1)+1=n-m+1-r=\widetilde{q}-r .
$$

If $q \mid n$ or $r=0$ then $\widetilde{q}-r=\widetilde{q}=\widehat{q}$; and if $q \nmid n$ or $r \geq 1$ then $\widetilde{q}-r \leq \widetilde{q}-1=\widehat{q}$. Hence if $t \leq m$ we have $t(q-1)+1 \leq \widehat{q} \leq \widetilde{q}$ and

$$
\widetilde{q}_{t}:=\min \{\widetilde{q}, t(q-1)+1\}=t(q-1)+1 \leq \widehat{q} .
$$

Therefore, by the theorem of Diederich-Fornaess, the lemma holds if $t \leq m$.

Next if $t \geq m+1$ and if the lemma holds for $1,2, \ldots, t-1$, then for any $k$ with $1 \leq k \leq t-1$ the family $\left\{D_{1}, \ldots, D_{t}\right\}$ satisfies the condition

$$
\begin{aligned}
& H^{j}\left(D_{i_{1}} \cap \cdots \cap D_{i_{k}}, \mathcal{F}\right)=0 \\
& \quad \text { for all } j \geq \widehat{q} \text { and all } i_{1}, \ldots, i_{k} \in\{1,2, \ldots, t\} .
\end{aligned}
$$

Hence by Proposition 1

$$
H^{j}\left(D_{1} \cap \cdots \cap D_{t}, \mathcal{F}\right) \cong H^{j+t-1}\left(D_{1} \cup \cdots \cup D_{t}, \mathcal{F}\right) \quad \text { if } \quad j \geq \widehat{q} .
$$


Notice here that if $t \geq m+1$ and $j \geq \widehat{q}$ then $j+t-1 \geq \widehat{q}+m \geq \widetilde{q}-1+m=n$.

Since the set $D_{1} \cup \cdots \cup D_{t}$ is open in $M$ and since $H^{n}(M, \mathcal{F})=0$ by assumption we have $H^{n}\left(D_{1} \cup \cdots \cup D_{t}, \mathcal{F}\right)=0$ (see Remark below). Therefore we obtain

$$
H^{j}\left(D_{1} \cap \cdots \cap D_{t}, \mathcal{F}\right)=0 \quad \text { if } \quad j \geq \widehat{q},
$$

which proves the lemma.

Theorem is the direct result of the above lemma and the theorem of Diederich-Fornaess (cf. [D-F], §5).

Remark. By the theorem of Greene-Wu ([G-W]), a connected complex manifold of dimension $n$ is $n$-complete if and only if it is noncompact. Therefore, if $D$ is noncompact complex manifold of dimension $n$ then by the theorem of Andreotti-Grauert $H^{n}(D, \mathcal{F})=0$ for any coherent analytic sheaf $\mathcal{F}$ on $D$. It is obvious that if $H^{n}(M, \mathcal{F})=0$ then $H^{n}(D, \mathcal{F})=0$ for any connected (and not necessarily noncompact) component $D$ of $M$.

\section{$\S 3 . \quad$ Example}

As in Section 2 we put $n=m q+r$. In $\mathbb{C}^{n}$, consider the complex linear subspaces defined by

$$
L_{i}:=\left\{\left(z_{1}, \ldots, z_{n}\right) \in \mathbb{C}^{n} \mid z_{(i-1) q+1}=\ldots=z_{i q}=0\right\}
$$

and put $D_{i}:=\mathbb{C}^{n} \backslash L_{i}$ for $i=1,2, \ldots, m$. Then each $D_{i}$ is $q$-complete but not $(q-1)$-complete (cf. [W]). If $q \nmid n$ or $r \geq 1$, we moreover put

$$
L_{m+1}:=\left\{\left(z_{1}, \ldots, z_{n}\right) \in \mathbb{C}^{n} \mid z_{m q+1}=\ldots=z_{n}=0\right\}
$$

and $D_{m+1}:=\mathbb{C}^{n} \backslash L_{m+1}$. Then $D_{m+1}$ is $r$-complete and particularly $q$ complete because $r<q$.

The number $\widehat{q}_{t}$ in Theorem is best possible for any $(n, q, t)$, where

$$
\widehat{q}_{t}:=\min \{\widehat{q}, t(q-1)+1\}= \begin{cases}t(q-1)+1 & \text { if } t \leq m \\ \widehat{q} & \text { if } t>m\end{cases}
$$

and

$$
\widehat{q}:=n-\left[\frac{n-1}{q}\right]=\left\{\begin{array}{lll}
n-m+1 & \text { if } & q \mid n \\
n-m & \text { if } & q \nmid n .
\end{array}\right.
$$

In fact, we have the following. 
EXAMPLE. Under the above notations, $H^{t(q-1)}\left(D_{1} \cap \cdots \cap D_{t}, \mathcal{O}\right) \neq 0$ for $t=1,2, \ldots, m$. Moreover, $H^{n-m-1}\left(D_{1} \cap \cdots \cap D_{m+1}, \mathcal{O}\right) \neq 0$ if $q \nmid n$.

In the example above, $\mathcal{O}$ denotes the sheaf of germs of holomorphic functions on $\mathbb{C}^{n}$. The example is a part of the following.

Proposition 2. Let $\alpha_{0}, \alpha_{1}, \ldots, \alpha_{t}$ and $n_{0}$ be integers such that $0=$ $\alpha_{0}<\alpha_{1}<\cdots<\alpha_{t}=n_{0} \leq n$. In $\mathbb{C}^{n}$, consider the complex linear subspaces defined by

$$
L_{i}:=\left\{\left(z_{1}, \ldots, z_{n}\right) \in \mathbb{C}^{n} \mid z_{\alpha_{i-1}+1}=z_{\alpha_{i-1}+2}=\cdots=z_{\alpha_{i}}=0\right\}
$$

and put $D_{i}:=\mathbb{C}^{n} \backslash L_{i}$ for $i=1,2, \ldots, t$. Then

$$
\left\{\begin{array}{l}
H^{n_{0}-t}\left(D_{1} \cap \cdots \cap D_{t}, \mathcal{O}\right) \neq 0 \\
H^{j}\left(D_{1} \cap \cdots \cap D_{t}, \mathcal{O}\right)=0
\end{array} \quad \text { if } \quad j \geq n_{0}-t+1 .\right.
$$

Proof. Since codim $L_{i} \leq n_{0}-(t-1)$ each $D_{i}$ is at least $\left(n_{0}-t+1\right)$ complete. Hence if we put $p:=n_{0}-t+1$ then $H^{j}\left(D_{i}, \mathcal{O}\right)=0$ for all $j \geq p$ and all $i$ with $1 \leq i \leq t$.

We shall now prove by induction that for any $k$ with $1 \leq k \leq t-1$ the family $\left\{D_{1}, \ldots, D_{t}\right\}$ satisfies the condition

$C(k, p)$

$$
H^{j}\left(D_{i_{1}} \cap \cdots \cap D_{i_{k}}, \mathcal{O}\right)=0
$$

$$
\text { for all } j \geq p \text { and all } i_{1}, \ldots, i_{k} \in\{1,2, \ldots, t\} \text {. }
$$

First $\left\{D_{1}, \ldots, D_{t}\right\}$ satisfies $C(1, p)$. Next if it satisfies $C(k-1, p)$ where $k \geq 2$, it follows from Proposition 1 that

$$
H^{j}\left(D_{i_{1}} \cap \cdots \cap D_{i_{k}}, \mathcal{O}\right) \cong H^{j+k-1}\left(D_{i_{1}} \cup \cdots \cup D_{i_{k}}, \mathcal{O}\right) \quad \text { if } \quad j \geq p .
$$

Since $D_{i_{1}} \cup \cdots \cup D_{i_{k}}=\mathbb{C}^{n} \backslash\left(L_{i_{1}} \cap \cdots \cap L_{i_{k}}\right)$ and since codim $\left(L_{i_{1}} \cap \cdots \cap L_{i_{k}}\right) \leq$ $n_{0}-(t-k)=p+k-1$, the set $D_{i_{1}} \cup \cdots \cup D_{i_{k}}$ is at least $(p+k-1)$-complete. Hence for any $k$ with $1 \leq k \leq t-1$ we have

$$
H^{j}\left(D_{i_{1}} \cap \cdots \cap D_{i_{k}}, \mathcal{O}\right)=0 \quad \text { if } \quad j \geq p,
$$

which implies that $\left\{D_{1}, \ldots, D_{t}\right\}$ satisfies $C(t-1, p)$.

Therefore, by Proposition 1 we obtain

$$
\begin{aligned}
& H^{j}\left(D_{1} \cap \cdots \cap D_{t}, \mathcal{O}\right) \cong H^{j+t-1}\left(D_{1} \cup \cdots \cup D_{t}, \mathcal{O}\right) \quad \text { if } \quad j \geq p \\
& H^{p-1}\left(D_{1} \cap \cdots \cap D_{t}, \mathcal{O}\right) \rightarrow H^{p+t-2}\left(D_{1} \cup \cdots \cup D_{t}, \mathcal{O}\right) .
\end{aligned}
$$


On the other hand,

$$
\left\{\begin{array}{l}
H^{n_{0}-1}\left(D_{1} \cup \cdots \cup D_{t}, \mathcal{O}\right) \neq 0 \\
H^{j}\left(D_{1} \cup \cdots \cup D_{t}, \mathcal{O}\right)=0 \quad \text { if } \quad j \geq n_{0}
\end{array}\right.
$$

because $D_{1} \cup \cdots \cup D_{t}=\mathbb{C}^{n} \backslash\left(L_{1} \cap \cdots \cap L_{t}\right)$ and $\operatorname{codim}\left(L_{1} \cap \cdots \cap L_{t}\right)=n_{0}$. Since $p:=n_{0}-t+1$ we thus obtain

$$
\left\{\begin{array}{l}
H^{n_{0}-t}\left(D_{1} \cap \cdots \cap D_{t}, \mathcal{O}\right) \neq 0 \\
H^{j}\left(D_{1} \cap \cdots \cap D_{t}, \mathcal{O}\right)=0 \quad \text { if } \quad j \geq n_{0}-t+1 .
\end{array}\right.
$$

This completes the proof of the proposition.

Acknowledgement. The author expresses her sincere thanks to the referee for the useful comment.

\section{REFERENCES}

[A-G] A. Andreotti and H. Grauert, Théorèmes de finitude pour la cohomologie des espaces complexes, Bull. Soc. Math. France, 90 (1962), 193-259.

[D-F] K. Diederich and J. E. Fornaess, Smoothing q-convex functions and vanishing theorems, Invent. Math., 82 (1985), 291-305.

[E-S] M. G. Eastwood and G. V. Suria, Cohomologically complete and pseudoconvex domains, Comment. Math. Helv., 55 (1980), 413-426.

[G-W] R. E. Greene and H. Wu, Embedding of open Riemannian manifolds by harmonic functions, Ann. Inst. Fourier (Grenoble), 25 (1975), 215-235.

[M-1] K. Matsumoto, Pseudoconvex domains of general order in Stein manifolds, Mem. Fac. Sci. Kyushu Univ., 43 (1989), 67-76.

[M-2] Boundary distance functions and q-convexity of pseudoconvex domains of general order in Kähler manifolds, J. Math. Soc. Japan, 48 (1996), 85-107.

[S-V] G. Sorani and V. Villani, q-complete spaces and cohomology, Trans. Amer. Math. Soc., 125 (1966), 432-448.

[W] K. Watanabe, Pseudoconvex domains of general order and vanishing cohomology, Kobe J. Math., 10 (1993), 107-115.

Department of Applied Mathematics

Osaka Women's University

Daisen-cho, Sakai 590-0035

Japan

kazuko@appmath.osaka-wu.ac.jp 